\title{
CHARACTERIZATION OF THE ROTATIONAL DYNAMICS OF MAGNETIC MICRODISCS IN SUSPENSION
}

\author{
N. Garraud" and D.P. Arnold \\ Interdisciplinary Microsystems Group, University of Florida, Gainesville, Florida, USA
}

\begin{abstract}
We present the fabrication and dynamic characterization of magnetic microdiscs suspended in liquid. The free-floating discs function like synchronized micro-shutters when actuated by an external magnetic field. Their motion is monitored via light transmission in response to a rotating magnetic field. We demonstrate that their dynamic rotation depends on the amplitude and the rotation frequency of the applied magnetic field. Consequently, the discs can function as local microsensors of their environment to optically study fluid viscosity change or particles/biological environment interactions.
\end{abstract}

\section{INTRODUCTION}

As depicted in Fig. 1, low-aspect-ratio ( micron in diameter, tens of nanometer thick) soft ferromagnetic discs exhibit a unique magnetic ground state, known as a "spin vortex" [1]. A key advantage of this closed magnetic spin arrangement is that the discs exhibit near-zero magnetic remanence and hence do not agglomerate with each other when in free suspension. With particle volumes $10,000 \times$ larger than superparamagnetic nanoparticles and the ability to impart large torques, these microdiscs are of extreme interest for tagging, trapping, actuating, or interrogating biological samples [2][3]. However, their dynamic response in a fluidic/biological environment is largely unknown, hence motivating this work.

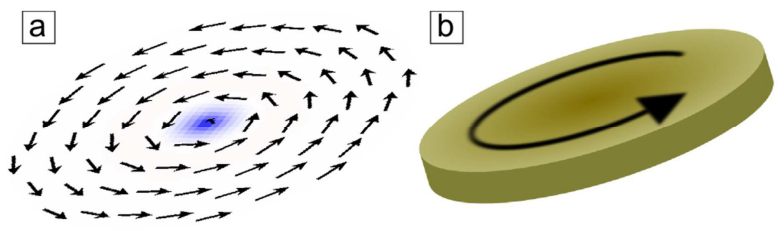

Figure 1: Magnetic spin vortex discs present no net remanence because of their closed magnetization configuration. (a) Magnetization ground state simulated by finite difference with OOMMF [4] (each arrow represents a magnetic moment). (b) Representation of the internal closed magnetization.

When subjected to an external magnetic field $\overrightarrow{B_{\text {ext }}}$, a magnetic structure will tend to rotate to align its easy-axis with the field. For soft magnetic discs with a small thickness $t$ compared to their diameter $\emptyset$, the easy-axis is in-plane. Hence, an ensemble of discs in suspension will operate synchronously and can function as an optical light shutter. In a quasi-static mode, when the magnetic field is aligned with the light path, the transmitted light is maximal (Fig. 2a). However, when the magnetic field is transverse, the discs act as a shutter and lower the transmitted light intensity (Fig. 2b).

This general optical shutter behavior has been previously demonstrated by Vitol, et al. [5]. They explored two different ways to enact rotation of the discs in suspension: a single-axis and a 2-axis coil system supplied with square wave currents. The 2axis system was shown to better control the discs orientation over a larger frequency range. In this article we use a similar 2-axis coil setup, but excited by sinusoidal current waveforms rather than square waves. The resulting B-field is a well-controlled rotating field, with constant amplitude and rotational velocity.
This approach gives us the opportunity to monitor both the light modulation amplitude and its phase shift. Unlike the amplitude, the phase is independent of the overall light intensity and the disc density/concentration. This overcomes many practical experimental challenges in quantifying the dynamics of the discs. As will be shown, at steady state, and for a given magnetic field amplitude, the phase shift can be derived from the balance between the magnetic torque and the hydrodynamic torque (Fig. 3a), each of which depends on the disc and fluid parameters, like the disc dimensions and the fluid viscosity. Hence monitoring of the phase shift can provide a valuable method for interrogation of one or more of these properties.
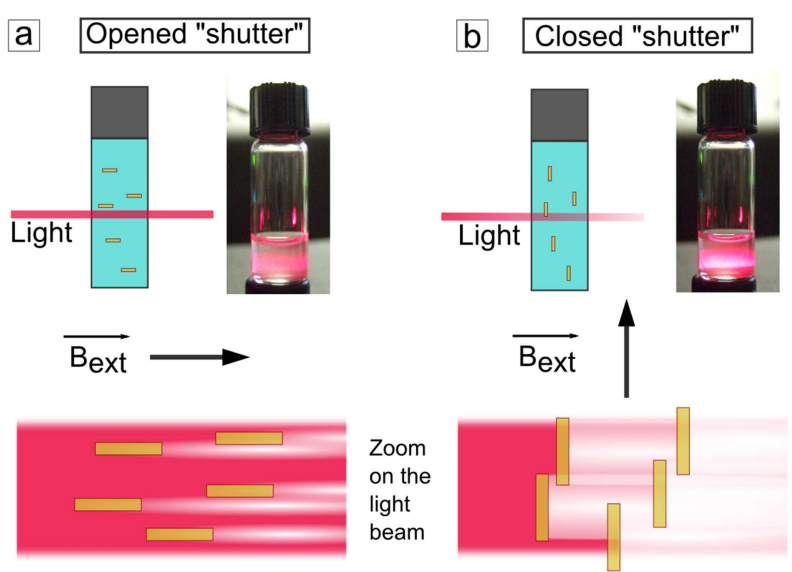

Figure 2: Light transmission modulation principle: the freefloating magnetic microdiscs in suspension rotate together and act as a shutter. The transmitted light is (a) maximal when the B-field is aligned to the beam axis and (b) minimal when the B-field is perpendicular to the beam axis.
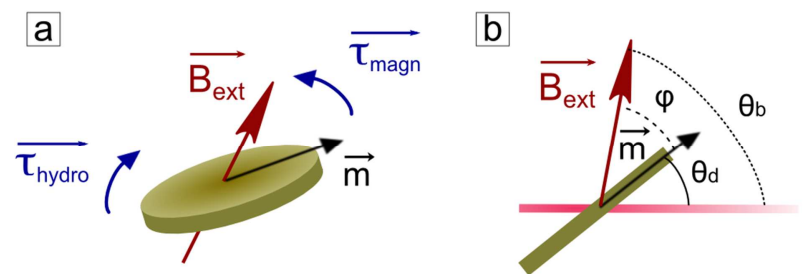

Figure 3: (a) The microdisc in a magnetic field, subjected to the magnetic torque and the hydrodynamic torque. (b) Definition of parameters used in the dynamic model.

\section{THEORY ON THE DISC MAGNETIC ACTUATION}

The external magnetic field $\overrightarrow{B_{\text {ext }}}$ modulated at $\omega=2 \pi f$ makes an angle $\theta_{b}=\omega t$ with the light path, whereas a disc makes an angle $\theta_{d}$ with respect to the light path (Fig. 3b). The angle difference between the disc plane and the magnetic field is the phase shift $\varphi$. Due to their small size dispersion, all the discs are assumed to have the same magnetic and mechanical behavior. Therefore, the recorded light intensity $I_{\text {meas }}$ depends on $\theta_{d}=$ $\omega t-\varphi$ :

$$
I_{\text {meas }}=I_{0}-I_{\text {block }}=I_{0}(1-\Delta \mathrm{I})=I_{0}\left(1-\delta\left|\sin \theta_{d}\right|\right)
$$

$I_{0}$ is the measured light without any discs in the solution, which 
depends on the optical system. $\Delta I$ is the percentage of light blocked by the discs in suspension and depends on the projection of the disc area in a plane perpendicular to the light path, the disc density in the solution, and the length of the light path. For one mechanical revolution of the discs, there are two variations in intensity (mathematically represented by the rectified sine term), and hence the light is modulated at a double frequency $2 f$.

The results are analyzed by studying the in-phase $\Delta I^{\prime}$ and the out-of-phase $\Delta I^{\prime \prime}$ components of the signal variation $\Delta I$, defined by:

$$
\left\{\begin{array}{l}
\Delta I^{\prime}=\delta \cos \varphi \\
\Delta I^{\prime \prime}=\delta \sin \varphi
\end{array}\right.
$$

\section{Phase shift dependency}

By neglecting the angular inertia torque (which is assumed to be small), the equation of motion of a magnetic disc in a uniform magnetic field is given by the equilibrium of the magnetic and the hydrodynamic torques:

$$
\overrightarrow{\tau_{\text {magn }}}+\overrightarrow{\tau_{\text {hydro }}}=\overrightarrow{0}
$$

The magnetic field is chosen low enough to operate in the linear domain described by the initial susceptibility $\chi_{0}$. Moreover, as shown in [6], the magnetic moment $\vec{m}$ of a flat vortex disc remains mainly in-plane, due to its high shape anisotropy, even when $\overrightarrow{B_{\text {ext }}}$ presents an out-of-plane component.

The magnetic moment is given by

$$
|\vec{m}|=\operatorname{Vol} \chi_{0}\left|\overrightarrow{H_{\text {ext }}}\right|_{\text {in-plane }}=\frac{\pi \emptyset^{2} t}{4 \mu_{0}} \chi_{0}\left|\overrightarrow{B_{\text {ext }}}\right| \cos \varphi
$$

The magnetic torque is thus calculated as

$$
\overrightarrow{\tau_{\text {magn }}}=\vec{m} \times \overrightarrow{B_{\text {ext }}}=\pi t \emptyset^{2} \chi_{0}\left|\overrightarrow{B_{\text {ext }}}\right|^{2} \sin (2 \varphi) / 8 \mu_{0}
$$

Due to its dependency on $2 \varphi$, the torque is null when the B-field is in-plane and out-of-plane with respect to the $\operatorname{disc}(\varphi=0$ or $\varphi=\pi / 2$ ), and reaches a maximum for $\varphi=\pi / 4$.

The hydrodynamic torque is given by:

$$
\left|\overrightarrow{\tau_{\text {hydro }}}\right|=-\eta K_{r} \dot{\theta_{d}}
$$

with $\eta$ the fluid viscosity and $K_{r}$ the rotational resistance depending on the disc dimensions and aspect ratio [7].

By solving (3), the rotational dynamics of the discs in suspension are governed by a critical frequency limit

$$
\omega_{\text {lim }}=\left|\overrightarrow{B_{\text {ext }}}\right|^{2} \frac{\pi \emptyset^{2} t \chi_{0}}{8 \mu_{0} \eta K_{r}}
$$

This limit defines two distinct frequency ranges:

i. $\quad \omega<\omega_{\text {lim }}$ : The discs rotate synchronously with $\overrightarrow{B_{\text {ext }}}$. A stable steady-state solution is found for $0<\varphi<\pi / 4$ :

$$
\varphi=\frac{1}{2} \arcsin \left(\frac{\omega}{\omega_{\text {lim }}}\right)
$$

ii. $\quad \omega>\omega_{\text {lim }}$ : The discs rotate asynchronously with $\overrightarrow{B_{\text {ext }}}$ and $\pi / 4<\varphi<\pi / 2$.

It is noted from (8) that the phase shift depends mainly on the magnetic field parameters $\left(\omega\right.$ and $\left|\overrightarrow{B_{\text {ext }}}\right|$ ) and the magnetic suspension characteristics (the fluid viscosity and the disc sizes and magnetic properties), which can be identified. It is also noted from (7) that the amplitude of the magnetic field can be adjusted to change $\omega_{\text {lim }}$.

Later experimental studies focus on the influence of the magnetic field frequency and magnitude on the phase shift and the light amplitude change.

\section{EXPERIMENTAL SETUP}

\section{Microdisc fabrication and characterization}

The microdiscs are microfabricated on a silicon substrate using a metal lift-off procedure (Fig. 4). Three layers are spin coated: a 200-nm thick PMMA sacrificial layer (A4 MicroChem), a 300-nm thick LOR 3A lift-off layer (MicroChem), and an 800nm thick S1813 photosensitive layer (Shipley, Microposit). The resist layers are patterned with a dense array of dots by direct-write UV laser at $405 \mathrm{~nm}$ (DWL 66fs Heidelberg) and developed to obtain the photoresist mask. A 70-nm-thick permalloy $\left(\mathrm{Ni}_{80} \mathrm{Fe}_{20}\right)$ layer is then deposited through the mask by magnetron sputtering followed by metal lift-off in AZ400K (AZ Electronic Materials).

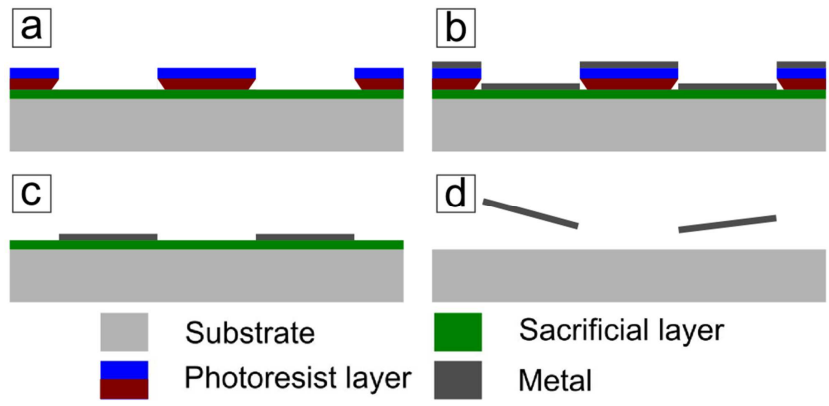

Figure 4: Microfabrication process: (a) Photoresist mask fabrication on a sacrificial layer. (b) 70-nm thick permalloy $\left(\mathrm{Ni}_{80} \mathrm{Fe}_{20}\right)$ sputtering. (c) Metal lift-off. (d) Discs releasing in water.

A dense array of 2.5- $\mu$ m-diameter, 70-nm-thick magnetic microdiscs is obtained on the sacrificial layer (Fig. 5a). Figure 6 shows the dramatic change of the magnetic behavior between a separately fabricated blanket permalloy layer (in blue) and the patterned array of microdiscs (in orange): the relative remanence drops from $20 \%$ to $0 \%$. By dissolving the sacrificial PMMA layer in acetone and by rinsing it in deionized water, 30 million discs are released into $1 \mathrm{~mL}$ of water (Fig. $5 \mathrm{~b}$ ).

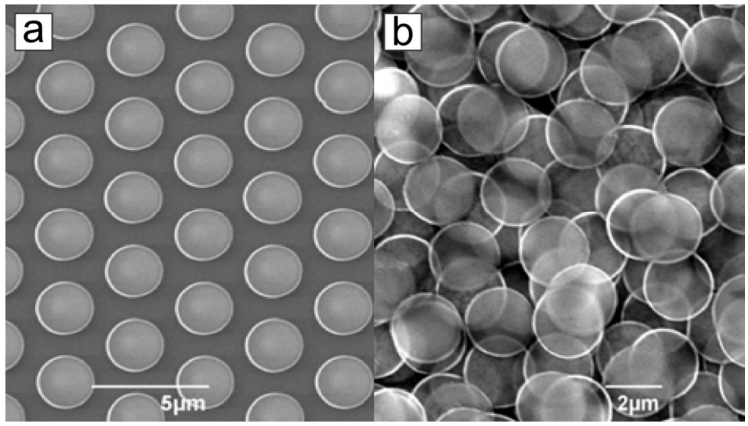

Figure 5: SEM pictures: (a) Array of 2.5- $\mu \mathrm{m}$ diameter, 70- $\mathrm{nm}$ thick magnetic microdiscs on the sacrificial layer. (b) Released discs on a substrate after the sacrificial layer dissolution.

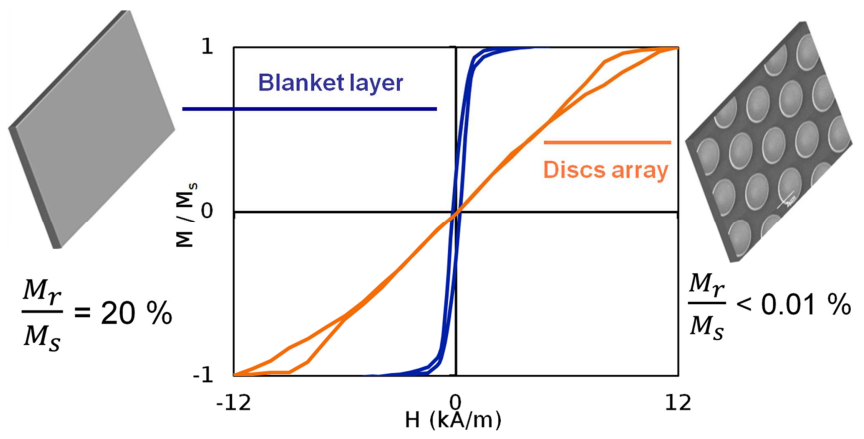

Figure 6: Magnetic characterization: in blue, the hysteresis loop of a 70-nm thick permalloy blanket layer; in orange, the behavior of spin vortex discs, showing no remanence. 


\section{Optical interrogation stage}

Inspired by [5], the experimental stage combines magnetic actuation with optical detection via a laser and photodiode (Fig. 7). A rotating magnetic field is created by a Helmholtz coil pair and an orthogonally oriented solenoidal coil, on top of which sits a glass vial with the disc suspension (Fig. 8). The Helmholtz pair and solenoid are supplied by two sinusoidal current waveforms in quadrature (frequency $f$ ), where the amplitude of each waveform is adjusted so as to supply equal field intensity on the sample. The volume of the disc suspension interacting with the light beam $\left(2 \times 5 \times 10 \mathrm{~mm}^{3}\right)$ is small enough that the discs are subjected to a spatially uniform rotating magnetic field up to $4 \mathrm{mT}$. Together the discs, rotating coherently, act as an optical shutter modulating the transmission path of a 650-nm, 5-mW laser. The transmitted light is measured by a photodiode, and the output signal is characterized by the DC component $I_{0}$, the relative AC amplitude $\Delta I$ and the phase shift $\varphi$, measured on an oscilloscope.

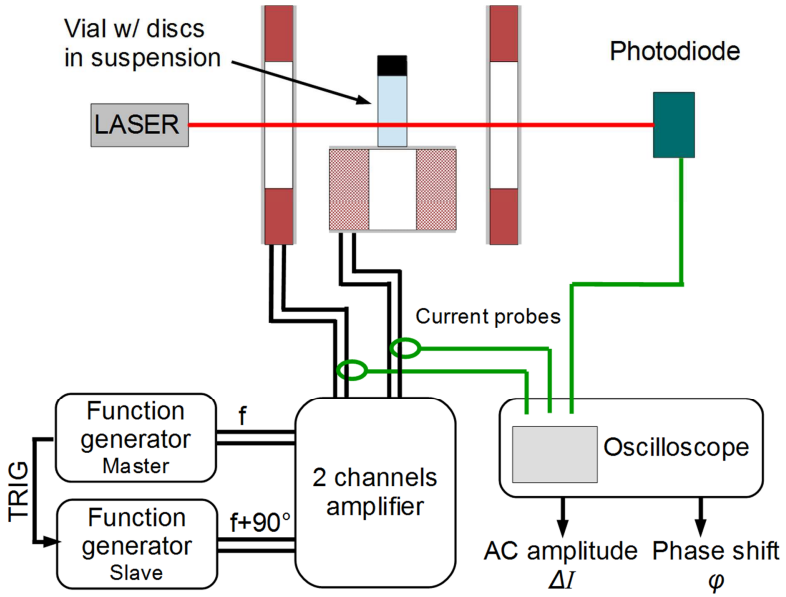

Figure 7: The experimental setup combines two orthogonal coils, a laser, and a photodiode. The coils are supplied with two sinusoidal current waveforms in quadrature, and all signals are monitored by an oscilloscope.

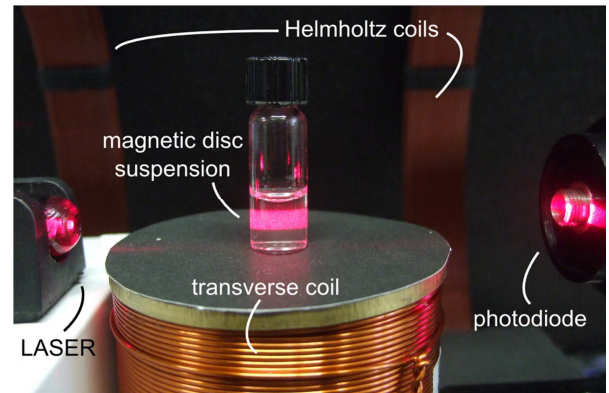

Figure 8: The magnetic actuation is made of a Helmholtz coil pair (parallel to the light path) and a transverse coil, which working together create a homogeneous rotating field in the vial space.

Figure 9 shows an example time-waveform of the light intensity modulation for $f=10 \mathrm{~Hz}$ in water. While varying the frequency, the electrical impedances of the coils are changing. As a consequence, the amplitudes of the two current waveforms in quadrature (Fig. 9a) are always controlled by changing the input currents, so the amplitude of the magnetic field is constant (Fig. $9 \mathrm{~b})$. The measured light is, as expected, a rectified sine modulated at $2 f=20 \mathrm{~Hz}$ and phase-shifted by $\varphi$ (Fig. 9c). a

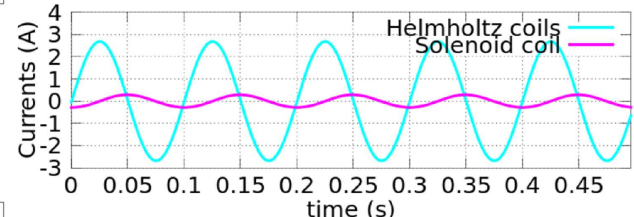

b

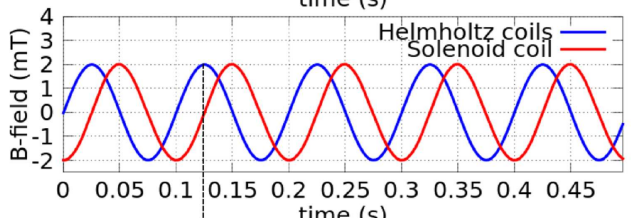

C

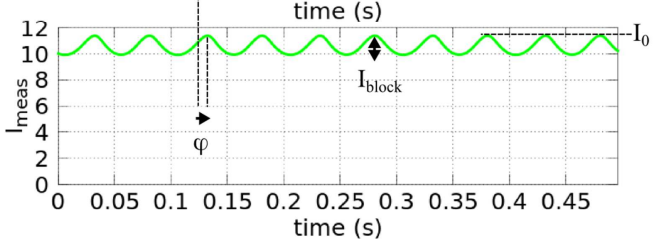

Figure 9: Example experimental results: (a) Recorded coil currents in quadrature at $f=10 \mathrm{~Hz} . \quad$ (b) Resulting rotating magnetic field. (c) Light intensity modulation at $2 f=20 \mathrm{~Hz}$.

Before dynamic experiments, the disc solution is sonicated for $5 \mathrm{~min}$ to disperse the discs into suspension. To investigate the overall stability of the experimental approach and the longevity of the discs in suspension, the light modulation parameters are measured for one hour (Fig. 10). Without any surfactant, the discs remain well suspended for at least one hour, allowing plenty of time for dynamic experiments.

a

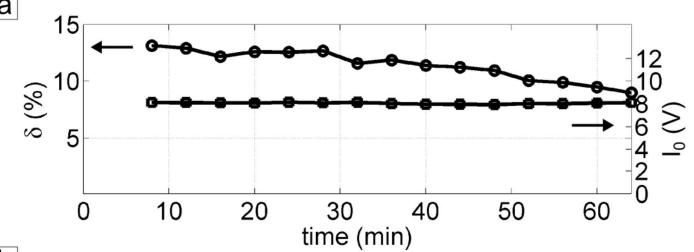

b

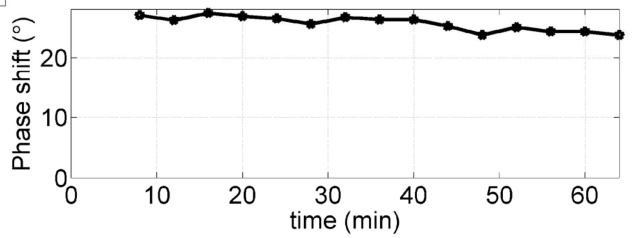

Figure 10: Suspension stability studied for 1 hour at $f=10 \mathrm{~Hz}$ under $1 \mathrm{mT}$. (a) Light modulation amplitude vs. time. (b) Phase shift vs. time.

\section{RESULTS}

\section{Effect of the modulation frequency}

Figure 11 shows the typical overall frequency response of the discs in suspension in water subjected to a $1 \mathrm{mT}$ rotating magnetic field. The amplitude and phase of $\Delta \mathrm{I}$ are presented in Fig. 11a. The real $\Delta I^{\prime}$ and imaginary $\Delta I^{\prime \prime}$ components are reported in Fig. $11 \mathrm{~b}$. Three frequency ranges are observed in Fig. 11 a. Below $10 \mathrm{~Hz}$, the phase shift is smaller than $\pi / 4$, and the relative variation of the light intensity decreases rapidly. At medium frequencies, between $10 \mathrm{~Hz}$ and $30 \mathrm{~Hz}$, the disc rotation is still synchronous with the external magnetic field. In this range, the intensity modulation amplitude $\Delta I$ is constant, and the phase continues to increase. Above the critical frequency $f_{\text {lim }}=40 \mathrm{~Hz}$, 
the viscous effects from the hydrodynamic torque lead to a decrease in amplitude and an increase in phase shift above $\varphi_{\text {lim }}=\pi / 4$. Fig. $11 \mathrm{~b}$ shows another key feature - a maximum in the imaginary part at $f_{0}=30 \mathrm{~Hz}$. This peak position, depending on the disc interactions with their environment, is a relevant parameter to extract to follow any change in the disc suspension (such as the viscosity).
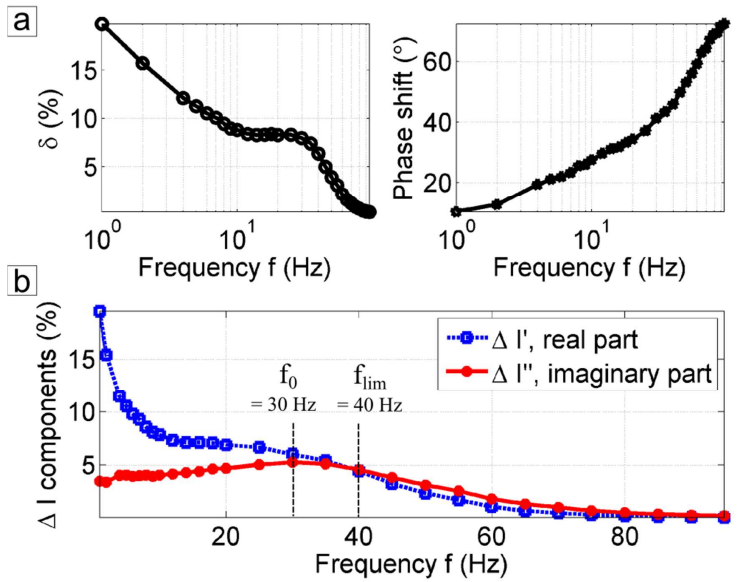

Figure 11: Frequency dependence of discs in suspension in water. (a) AC light amplitude and phase shift vs. magnetic field frequency. (b) Real and imaginary components projection. The imaginary peak is related to the discs environment properties.

The simple model developed in the theoretical section explains the observed behaviors as the synchronous $(10 \mathrm{~Hz}-$ $40 \mathrm{~Hz})$ and the asynchronous modes $(>40 \mathrm{~Hz})$ delimited by $\omega_{\text {lim }}$. However, further investigations are needed to model the disc suspension behavior at lower frequencies $(<10 \mathrm{~Hz}$ in this case).

\section{Effect of the magnetic field magnitude}

Figure 12 shows the dynamics response by changing the magnetic field magnitude over a range from 0.5 to $4 \mathrm{mT}$ at several different fixed frequencies.
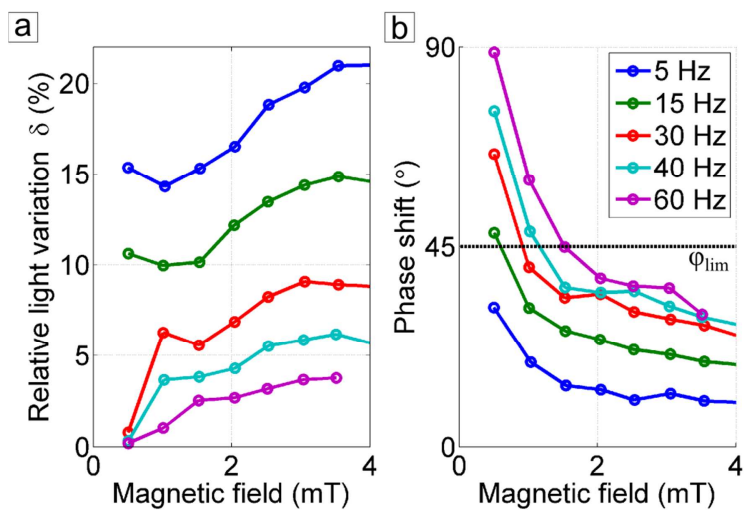

Figure 12: Magnetic field magnitude dependence of discs in suspension in water at several frequencies. (a) AC light amplitude and (b) phase shift vs. magnetic field magnitude.

The largest changes in $\delta$ and $\varphi$ occur for frequencies around $f_{0}$ and $f_{\text {lim }}$. At low frequencies (such as $5 \mathrm{~Hz}$ ), $\delta$ and $\varphi$ changes are smaller than for higher frequencies, since the phase shift never reaches the critical phase of $\pi / 4$. Therefore, changes in the rotational dynamics of the suspension due to the change of one environmental parameter can be studied by monitoring $\delta$ and $\varphi$ while doing an amplitude magnetic field sweep at frequencies around $f_{\text {lim }}$. Also, experimentally, changing the field amplitude presents the advantage to be much faster and easier than sweeping the frequency.

\section{CONCLUSION}

We present an optical interrogation methodology for studying the rotational dynamics of suspended magnetic microdiscs in response to a constant-amplitude rotating magnetic field. The system enables measurement of the phase shift as a function of the frequency and amplitude of the magnetic field. The phase shift measurement is particularly interesting because it is independent of experimental variables such as the disc density in solution and uncontrolled variations in intensity. The system functionality is demonstrated using $2.5-\mu \mathrm{m}$-diameter permalloy microdiscs. The dynamics of the discs above $10 \mathrm{~Hz}$ are explained by a simple model. However further modelling is needed to understand the dynamic behavior at lower frequencies.

In future work, measurements obtained with this setup will be coupled with parameter identification procedures and dynamic computational simulations (as done on magnetic ellipsoids [7]) to explore more complex models. Potential future applications include optical determination of viscosity or detection of specific molecule adsorption on the discs, which may alter the hydrodynamic diameter and hence dynamic properties.

\section{ACKNOWLEDGEMENT}

This work was supported in part by the UF Office of Sponsored Research. The authors acknowledge Dr. Carlos Rinaldi for his valuable technical discussion on the rotational dynamics of magnetic particles in suspension.

\section{REFERENCES}

[1] R.P. Cowburn, D.K. Koltsov, A.O. Adeyeye, M.E. Welland, and D.M. Tricker, "Single-domain circular nanomagnets", Physical Review Letters, 83, 1042 (1999).

[2] D-H. Kim, E.A. Rozhkova, I.V. Ulasov, S.D. Bader, T. Rajh, M.S. Lesniak, and V. Novosad, "Biofunctionalized magneticvortex microdiscs for targeted cancer-cell destruction", Nat. Mater., 9, 165 (2010).

[3] S. Leulmi, H. Joisten, T. Dietsch, C. Iss, M. Morcrette, S. Auffret, P. Sabon, and B. Dieny, "Comparison of dispersion and actuation properties of vortex and synthetic antiferromagnetic particles for biotechnological applications", Appl. Physics Letters, 103, 132412 (2013).

[4] M.J. Donahue, and D.G. Porter, "OOMMF User's Guide, Version 1.0", Interagency Report NISTIR 6376, National Institute of Standards and Technology (1999).

[5] E.A. Vitol, V.G. Yefremenko, S. Jain, J. Pearson, E.A. Rozhkova, S.D. Bader, and V. Novosad, "Optical transmission modulation by disk-shaped ferromagnetic particles", J. of Applied Physics, 111, 07A945-1 (2012).

[6] N. Garraud, and D.P. Arnold, "Experimental investigation of magnetic anisotropy in spin vortex discs", J. Applied Physics, 115, 17D128 (2014).

[7] J.H. Sánchez, and C. Rinaldi, "Rotational Brownian dynamics simulations of non-interacting magnetized ellipsoidal particles in d.c. and a.c. magnetic fields", J. of Magnetism and Magnetic Material, 321, 2985 (2009).

\section{CONTACT}

*N. Garraud, tel: +1-352-328-8556; ngarraud@ufl.edu 